\title{
L'anthropocène est un « accumulocène »
}

\section{Anthropocene is an « accumulocene »}

\section{JEAN-BAPTISTE FRESSOZ, CNRS, EHESS}

Résumé : Si les injonctions à amorcer une transition pour sortir d'une « crise environnementale » se font nombreuses, elle ne doivent pas faire oublier le passage plus fondamental à une nouvelle ère géologique d'origine humaine: l'anthropocène. Ce cadre d'analyse ne doit pas être cantonné au système terre mais doit être étendu au système technique et matériel: non seulement les flux de matière s'empilent dans les différents compartiments du système-terre, mais les flux matériels anthropogéniques eux-mêmes tendent à suivre historiquement une logique d'accumulation.

\begin{abstract}
Beyond the immediate threat of the current environmental crisis, biologists and physicists suggest that human activity has become substantial enough to modify the geological order, a phenomenon often referred to as the Anthropocene. While it is now widely recognized that the earth is deeply affected by the humans extractions and rejections of materials, it should also be noticed that the material flows fueled by the human economic activity themselves follow a cumulative historical process.
\end{abstract}

\section{Introduction}

Le principal mérite du concept d'anthropocène tient à sa dimension temporelle : il souligne avec force le caractère irréversible des phénomènes biogéochimiques planétaires enclenchés par l'agir humain. Contrairement à l'expression « crise environnementale » qui désigne une épreuve brève dont l'issue serait imminente, l'anthropocène nomme un point de non-retour, une bifurcation géologique dans l'histoire de la planète Terre, sans retour prévisible à la normale de l'Holocène ${ }^{1}$. Ce que nous vivons n'est pas une «crise environnementale », mais une révolution géologique d'origine humaine.

Le réchauffement climatique par exemple est un phénomène cumulatif : la temporalité du cycle géologique du carbone et l'inertie thermique des océans font que le $\mathrm{CO}_{2}$ émis par les entrepreneurs de la Révolution industrielle élèvent et élèveront longtemps encore le niveau des océans. L'inertie du système-terre est telle que les conséquences du capitalisme industriel réverbèreront sur le futur de la planète bien plus longtemps sans doute qu'il ne durera lui-même.

\footnotetext{
${ }^{1}$ L'époque géologique depuis la dernière glaciation il y a environ 12000 ans.
} 
Cette courte contribution propose d'étendre cette temporalité propre à l'anthropocène du système terre au système technique et matériel. L'anthropocène désigne une double irréversibilité, une double accumulation, un cumul de cumuls : non seulement les flux de matière s'empilent dans les différents compartiments du système-terre, mais les flux matériels anthropogéniques eux-mêmes tendent à suivre historiquement une logique d'accumulation.

$\mathrm{Au}$ départ, l'anthropocène était une interpellation des sciences du système terre à l'endroit des géologues. Il était temps, expliquait en substance Paul Crutzen (2002), prix de Nobel de Chimie pour ses travaux sur la couche d'ozone, que ses collègues officialisent l'entrée de la Terre dans une nouvelle époque marquée par l'arrivée de «l'homme» en tant que force planétaire dominante 2 . L'anthropocène était une incursion sur le terrain de la géologie et c'est ce qui explique l'étrangeté des débats qu'il a suscités quant à sa date de départ - les débuts de l'agriculture ? la révolution industrielle ? les années 1950? — et ses marqueurs - l'augmentation du CO2 dans les carottes glaciaires ? La bombe atomique ? Le plastique ? Le béton ? Ou bien les os de poulet ? Les géologues se retrouvent dans la position inconfortable de devoir imaginer les traces futures laissées par la perturbation de cycles biogéochimiques dans des terrains sédimentaires qui n'existent pas encore (Zalasiewicz et al., 2018) ${ }^{3}$.

Mais tout autant que la géologie, l'anthropocène interpelle aussi les sciences sociales. Les historiens et les économistes en particulier. Car c'est bien à eux qu'incombe la tâche d'étudier les causes, les facteurs, les dynamiques historiques faisant entrer la Terre dans une nouvelle époque. Face à l'anthropocène, la réaction naturelle des historiens fut de critiquer le retour de « l'espèce humaine » sous la forme d'un anthropos indifférencié, collectivement responsable des dérèglements $\mathrm{du}$ système terre. On retrouvait à deux siècles d'écart une vision malthusienne de la crise environnementale. D'où la multiplication d'alternatives visant à désigner des acteurs historiques un peu plus précis : « capitalocène », « anglocène », « thanatocène », « thermocène », « plantatiocène » etc $^{4}$. Mais pour que ces propositions ne soient pas de simples provocations, encore fallait-il connecter des catégories historiographiques classiques - la révolution industrielle, le capitalisme,

\footnotetext{
2 Paul J. Crutzen, « Geology of mankind », Nature, vol. 415, 3 janv. 2002

3 Jan Zalasiewicz et al., The Anthropocene as a Geological Time Unit, Cambridge, Cambridge University Press, 2018.

$4 \quad$ Qui attribuent un rôle géologique respectivement au capitalisme, aux puissances hégémoniques anglaise puis états-unienne, au technologies militaires destructrices, à la révolution thermoindustrielle, à l'impérialisme et à l'économie de plantation etc. Christophe Bonneuil et Jean-Baptiste Fressoz, L'Evénement Anthropocène. La Terre, l'histoire et nous, Paris, Le Seuil, 2013 ; Andreas Malm and Alf Hornborg, « The geology of mankind? A critique of the Anthropocene narrative ", The Anthropocene Review, 2014, vol. 1, n¹, p 62-69; Jason Moore, Capitalism in the web of life, Verso, 2015.
} 
les guerres, les empires, la globalisation — aux déterminants physiques de l'anthropocène et donc aux quantités de matières (aux giga-tonnes de carbone, de phosphore, d'azote etc.) qui modifient les cycles biogéochimiques. L'anthropocène (et sa critique) conduisaient ainsi à re-considérer l'histoire et l'économie d'un point de vue matériel, quantitatif et global.

D'où la pertinence dans ce débat des travaux issus de l'analyse des flux de matière (MFA pour Material Flow Analysis). Cette approche dynamique depuis les années 1990 consiste à utiliser les statistiques douanières, minérales et agricoles pour établir les tonnes de minéraux extraits du sous-sol, la biomasse récoltée, les importations et les exportations comptabilisées en poids et retracer ainsi les «profils métaboliques » des nations. Elle permet de quantifier avec précision les flux de matières qui alimentent une économie nationale, à la fois «l'extraction domestique » et la «balance commerciale physique» (les exportations défalquées des importations), toujours mesurées en tonnes. Face au système terre, plutôt qu'un anthropos on peut, grâce à ces travaux, faire figurer un système de flux de matières circulant au sein de l'économie mondiale ${ }^{5}$.

L'effort de re-matérialisation démontre le caractère inégalitaire des «systèmes mondes » : les nations riches consomment généralement plus de matières - que ce soit de leurs propres territoires ou de ceux des autres pays - que la moyenne. Grâce à la MFA il devient possible d'objectiver les relations métaboliques qu'entretiennent différentes nations avec la matière globale. Plusieurs métriques sont disponibles : calculer par exemple le déficit de la balance commerciale physique. Celle de la France est ainsi constamment déficitaire depuis le XIXe siècle avec un accroissement brutal après 1958 lié à l'augmentation spectaculaire des importations de pétrole à partir de cette date. De manière plus subtile, Alf Hornborg propose de rapporter les biens échangés aux «hectares fantômes » nécessaires pour les produire. Il a ainsi calculé qu'au milieu du XIXe siècle en échangeant 1000 livres sterling de textile fabriqué à Manchester contre 1000 livres sterling de coton brut produit aux Etats-Unis, l'Angleterre était gagnante à $46 \%$ en termes de travail incorporé (échange inégal) et à $6000 \%$ en termes d'hectares incorporés c'est à dire qu'il avait fallu 60 fois plus d'hectares pour produire le coton brut que pour nourrir les ouvriers

\footnotetext{
$5 \quad$ Le World Resource Institute (Washington) et l'Institute of Social Ecology (Vienne) ont été moteurs. A. Adriaanse, S. Bringezu, Y Moriguchi, Y. et al., Resource Flows : The Material Basis of Industrial Economies, World Resources Institute, Washington, 1997 ; E. Matthews, E. Bringezu, S.Fischer-Kowalski, M. et al. The Weight of Nations: Material Outflows from Industrial Economies, World Resources Institute, Washington, 2000. EUROSTAT a formalisé les méthodes de calcul de la MFA en $2001 \mathrm{cf}$. Economy-wide material flow accounts and derived indicators A methodological guide. Voir aussi Vaclav Smil, Making of the Modern World, Materials and dematerialization, Wiley, 2014. Avec Christophe Bonneuil, nous avons mis à profit ces travaux pour justifier la proposition de Capitalocène dans la seconde édition (2016) de l'Evénement Anthropocène.
} 
britanniques filant ce coton. Alf Hornborg (2013) parle à ce propos «d'échange écologique inégal $^{6} \gg$.

Point important et qui peut être contre-intuitif, l'échange écologique inégal tend à s'accroître au cours du XXe siècle. Il est beaucoup moins fort à l'époque des empires qu'à celle du néolibéralisme. L'Europe reposait davantage sur ses ressources propres (en énergie, en minerais et en bois) au XIXe siècle que de nos jours. Les grandes puissances du XIXe siècle se livrent certes au pillage colonial des ressources mais sont avant tout de grandes puissances extractivistes sur leur propre territoire. Dans la seconde moitié du XIXe siècle, le Royaume-Uni assurait l'approvisionnement en charbon d'un très grand nombre de pays pauvres sur le pourtour méditerranéen et jusqu'en Amérique du sud. Sa balance commerciale physique est alors excédentaire. Elle est devenue massivement déficitaire seulement dans l'après-guerre avec l'augmentation des importations de pétrole (Schandl et Schulz, 2002) ${ }^{7}$. De même, jusque dans les années 1960, les USA exportent beaucoup plus de matières (charbon, pétrole, produits agricoles, bois) qu'ils n'en importent (Gierlinger et Krausmann, ?) ${ }^{8}$. La France — qui a la particularité parmi les pays riches d'avoir une balance commercial physique déficitaire dès le XIXe siècle consommait moins d'une tonne de matière étrangère par an et par habitant en 1900 contre 6 tonnes en 2000. Les importations de matières représentent un peu plus de $10 \%$ de son extraction domestique en 1900 et $50 \%$ en 2000 (Magalhães et al., 2019) ${ }^{9}$. D’une manière générale, l'extraversion matérielle de l'Europe de l'Ouest et du Japon s'accroît brutalement dans la seconde moitié du XXe siècle avec l'explosion des importations de pétrole. Il est probable qu'à partir des années 1950 l'accroissement des échanges commerciaux encourage la relocalisation des activités extractives sur certains territoires et donc l'inégalité écologique des échanges internationaux (Eisenmenger et Giljum, 2010) 10.

\footnotetext{
6 Alf Hornborg, Global Ecology and Unequal Exchange. Fetishism in a Zero-Sum World, Londres, Routledge, 2013, p. 85-91. Il faudrait aussi remarquer qu'a contrario le Royaume-Uni exporte alors beaucoup de produits sidérurgiques, très intensifs en énergie, vers les USA.

$7 \quad$ Heinz Schandl et Niels Schulz, « Changes in the United Kingdom's natural relations in terms of society's metabolism and land-use from 1850 to the present day », Ecological Economics, vol. 41, 2002, p. $203-221$.

$8 \quad$ Sylvia Gierlinger and Fridolin Krausmann, « The Physical Economy of the United States of America Extraction, Trade, and Consumption of Materials from 1870 to 2005 », Journal of Industrial Ecology, Volume 16, Number 3 .

$9 \quad$ Nelo Magalhães et al. « The Physical Economy of France (1830-2015). The History of a Parasite? », Ecological Economics, vol. 157, March 2019, p. 291-300.

$10 \quad$ Nina Eisenmenger et Stefan Giljum, « Evidence from Societal Metabolism Studies for Ecological Unequal Trade », in A. Hornborg et C. Crumley (eds.), The World System and the Earth System, Walnut Creek, Left Coast Press, 2010 .
} 
Les analyses en termes de flux de matières construisent donc une vision nouvelle de l'histoire du dernier siècle, une histoire à la fois matérielle, quantitative et globale. Elles révèlent son caractère inégalitaire et elles révèlent aussi sa nature fondamentalement cumulative. Ce faisant elle prend à rebrousse-poil les historiens habitués à réfléchir en termes de " transitions ${ }^{11}{ }$. Le phénomène —assez déprimant— que révèlent les analyses MFA est qu'à l'échelle globale, les matières n'ont, pour l'instant, fait que s'additionner. Toutes les «transitions » n'ont pour l'instant jamais produit de soustractions matérielles. Cela vaut surtout pour les matériaux de construction (le béton pèse très lourd dans ces calculs), les combustibles fossiles, les minerais, mais aussi pour la biomasse dont la consommation globale a doublé entre 1900 et 2000 (Krausman et al., 2013) ${ }^{12}$. Les guerres, les crises économiques ou les pandémies ne font en général qu'une petite encoche dans une courbe de la consommation matérielle globale qui est toujours ascendante depuis (au moins) un siècle.

Comment expliquer ce phénomène ? Prenons par exemple le bois dont la consommation globale n'a fait que croître depuis la révolution industrielle. Une première raison, évidente mais qui n'est pas la plus importante, tient aux usages qui perdurent dans les pays pauvres. Le bois reste la première source d'énergie « renouvelable» dans le monde pauvre : 3 milliards de personnes qui sont aussi les principales victimes de la pollution atmosphérique, brûlent du bois pour leur cuisine et leur chauffage. L'urbanisation en Afrique n'a pas conduit à une disparition du bois énergie mais à une substitution du bois vers le charbon de bois dont la consommation a été multipliée par 6 entre 1960 et 2010 (Zulu et Richardson, 2012) ${ }^{13}$. Mais ce sont surtout les réorientations d'usage qui compensent ou sur-compensent les substitutions. Par exemple si au XIXe siècle l'usage du bois de feu recule dans les pays industriels face au charbon, la consommation de bois croit très fortement pour d'autres besoins, principalement la construction, le papier, le carton et la cellulose. Si le charbon se substitue au bois-énergie il entre aussi en « symbiose » avec le bois-matière par exemple pour le boisage de mines, la construction des chemins de fer, la production de tonneaux et de caisses liés au développement du commerce ${ }^{14}$.

\footnotetext{
11 Je pense surtout à l'histoire de l'énergie constamment abordé à travers la notion de transition. L'histoire globale de l'énergie n'est pas celle de «transitions »—ou alors il faudrait définir transition dans le sens vague et sans intérêt pour la question climatique de changement relatif d'importance des sources d'énergie primaire.

12 Fridolin Krausman et al. « From resource extraction to outflows 1900-2015 », Global environmental change, 2018. On passe de $13 \%$ de toute la biomasse produite consommée par les humains en 1910 à $25 \%$ en 2005 . Cf. Krausmann et al., « Global human appropriation of net primary production doubled in the 20th century », PNAS, 2013, vol. $110, \mathrm{n}^{\circ} 25$, p. 10324-10329.

13 Leo Zulu, Robert B. Richardson, « Charcoal, livelihoods, and poverty reduction: Evidence from sub-Saharan Africa », Energy for sustainable development, 2012, 17-2, p. 127-137.

$14 \quad$ Egon Glesinger, Le bois en Europe, Paris, Sirey, 1932.
} 
Un autre facteur est que les nouvelles matières premières alimentent de nouveaux usages davantage qu'elles ne servent à satisfaire des besoins anciens. L'exemple phare est ici le pétrole qui dans la première moitié du XXe siècle ne se substitue pas — ou qu'à la marge — au charbon mais sert surtout à faire avancer des voitures. Si l'usage du charbon décroît relativement au pétrole, il reste que sa consommation croît continûment au XXe siècle, et que globalement, on n'en a jamais autant brûlé qu'en 2018. La grande innovation énergétique du XXe siècle, à savoir l'électricité, renforce sa centralité. On assiste à partir des années 1970 à une croissance très importante de la production d'électricité à partir de charbon en Chine évidemment mais aussi dans des pays parmi les plus riches au monde. C'est en 2008 que la consommation de charbon des Etats-Unis atteint son maximum historique. Le charbon est tout autant l'énergie de la « révolution internet » du début du XXIe siècle (un réseau d'électrons supplémentaire) que celle de la mal nommée « révolution industrielle » du XIXe siècle. Pour le moment, le nucléaire et les renouvelables n'ont pas réussi à faire diminuer les fossiles dans le mix énergétique mondial qui demeure fondamentalement carboné (de l'ordre de $80 \%$ de l'énergie primaire globale).

Si l'énergie focalise l'attention depuis les années 1970, elle n'est absolument pas exceptionnelle. Au cours des deux derniers siècles, l'éventail des matières premières utilisées s'élargit sans cesse et chacune des matières est consommée en quantités croissantes. Les processus de substitutions sont largement compensés par les effets rebonds ${ }^{15}$ et par les réorientations d'usage. Entre 1900 et 2015, le poids total des matières premières consommées par l'économie mondiale a été multipliée par 12 (Krausman et al., 2018). ${ }^{16}$ Depuis la seconde guerre mondiale, malgré la prolifération des produits de synthèse (plastiques etc.) aucune grande matière première n'a décru, hormis la laine de mouton qui recule face aux fibres synthétiques, ce qui n'est d'ailleurs pas une bonne nouvelle pour l'environnement. Entre 1960 et 2010, sur les 69 principales matières premières, seules six ont vu leur consommation mondiale décroitre. Et pour cinq d'entre elles (amiante, mercure, beryllium, tellurium et thallium) cette décroissance est due à leur toxicité et à des interdictions nationales (Magee et al. 2017) ${ }^{17}$. Durant le dernier demi-siècle, croissance et innovation n'ont encore jamais produit de véritable substitution à l'échelle globale. Dit autrement : nulle matière première n'est encore devenue obsolète.

\footnotetext{
15 L'effet rebond caractérise l'augmentation de la consommation liée à une amélioration de l'efficacité énergétique d'un produit et peut in fine conduire à une augmenation des dégradations environnementales.

16 Krausman et al. « From resource extraction to outflows 1900-2015 », Global environmental change, 2018

17 Christopher L. Magee Tessaleno C. Devezas, « A simple extension of dematerialization theory: Incorporation of technical progress and the rebound effect », Technological Forecasting \& Social Change, vol. 117, 2017, p. 196-205.
} 
$\mathrm{Au}$ XXe siècle, les guerres, les crises économiques et les pandémies (celle de 1918 par exemple) n'ont jamais réussi qu'à ralentir temporairement la dynamique d'accumulation matérielle globale. Ces deux dernières décennies, malgré la crise environnementale et malgré la crise financière, celle-ci s'est même accélérée : entre 2002 et 2015, en à peine 13 ans, on a extrait 1000 Gt de matières du sol, soit un tiers de tout ce qui avait été extrait depuis 1900. On assiste donc depuis 2002 à une nouvelle accélération de la consommation matérielle bien plus forte que la fameuse «grande accélération》 des années 1950. La pandémie de COVID 19 et la récession économique actuelle nourrissent l'espoir du pic : pic du charbon, pic du pétrole, pic du béton et peut être celui d'un « peak-all », d'une décrue de la consommation globale de matière. Mais l'histoire ne rend malheureusement guère optimiste sur ce point.

Bibliographie :

Paul J. Crutzen, « Geology of mankind », Nature, vol. 415, 3 janv. 2002

LEO Zulu, RoBert B. RichaRdson, «Charcoal, livelihoods, and poverty reduction: Evidence from sub-Saharan Africa », Energy for sustainable development, 2012, 17-2, p. 127-137.

EgON GLESINGER, Le bois en Europe, Paris, Sirey, 1932.

KRAUSMAN ET AL. « From resource extraction to outflows 1900-2015 », Global environmental change, 2018

Fridolin Krausman et AL. «From resource extraction to outflows 1900-2015 ", Global environmental change, 2018.

Krausmann et al., "Global human appropriation of net primary production doubled in the 20th century ", PNAS, 2013, vol. 110, n²5, p. 10324-10329.

Sylvia Gierlinger and Fridolin Krausmann, «The Physical Economy of the United States of America Extraction, Trade, and Consumption of Materials from 1870 to 2005 ", Journal of Industrial Ecology, Volume 16, Number 3.

Nelo Magalhães et Al. «The Physical Economy of France (1830-2015). The History of a Parasite?», Ecological Economics, vol. 157, March 2019, p. 291-300.

Nina Eisenmenger et STEFan GiLJum, «Evidence from Societal Metabolism Studies for Ecological Unequal Trade ", in A. Hornborg et C. Crumley (eds.), The World System and the Earth System, Walnut Creek, Left Coast Press, 2010.

Alf Hornborg, Global Ecology and Unequal Exchange. Fetishism in a Zero-Sum World, Londres, Routledge, 2013, p. 85-91.

HeINZ SCHANDl ET NiEls SChUlz, «Changes in the United Kingdom's natural relations in terms of society's metabolism and land-use from 1850 to the present day ", Ecological Economics, vol. 41, 2002, p. 203-221.

Jan Zalasiewicz et al., The Anthropocene as a Geological Time Unit, Cambridge, Cambridge University Press, 2018.

Christophe Bonneuil Jean-Baptiste Fressoz, L'Evénement Anthropocène. La Terre, l'histoire et nous, Paris, Le Seuil, 2013 